\title{
Alteration of composite resin after the application of fluoride varnish: an in vitro study
}

\begin{abstract}
Introduction: The characteristics of the surface in the restorative material are fundamental for its longevity, but some chemical solutions may be able to damage them, such as fluoride varnish, generating biofilm accumulation and discoloration of the material.
\end{abstract}

Aim: To compare the surface roughness and microhardness of the composite resin after application of the fluoride varnish.

Materials and methods: The total sample was 144 specimens. The roughness and microhardness test were measured four times. The ANOVA statistical test was performed for repeated samples according to the results of the Kolmogorov-Smirnov normality test. The present study presented a confidence level of $95 \%$ and $\mathrm{p}<0.05$.

Results: The group fluoride of sodium with tri-calcium phosphate had an increase of microhardness after the applications; likewise it showed higher roughness in comparison to the varnishes studied.

Conclusion: The varnish with sodium fluoride with tri-calcium phosphate increases the surface microhardness of the restorative material, however it increases the roughness of the surface; on the other hand, varnishes with sodium fluoride and sodium fluoride with CPP$\mathrm{ACP}$ reduce both the microhardness and the roughness of the resin.

Keywords: topical fluorides, dental restoration, hardness test, restorative materials, discoloration
Volume 10 Issue 5 - 2019

\author{
Denisse Aguilar G, Paola Hashimoto V \\ Department of Pediatric Dentistry, Scientific University \\ of the South, Peru
}

Correspondence: Denisse Aguilar G, Department of Pediatric Dentistry, Scientific University of the South, Peru,

Email daguilar@cientifica.edu.pe

Received: October 17, 2019 | Published: October 24, 2019

\section{Introduction}

Restorative dental materials are used in daily clinical practice due to the aesthetics it provides and biocompatibility with the dental surface, thus allowing their preservation. ${ }^{1}$

The characteristics of the surface have a fundamental role in the longevity of restorations, because of the biodegradation caused by chemical solutions or even preventive means, such as oral hygiene products and fluoride varnish can be able to damage the surface of restorative materials, causing accumulation of biofilm, wear and discoloration..$^{2-4}$

In spite of the measures taken, with the disruption of enamel and dentin it is necessary to use a restorative material to attend the progression of the caries disease. The decision of the material varies according to the type of lesion, cavity size, working protocol, among others. However, the difference is the use of fluoride varnish during treatment, which due to its properties it would be understood that it could lengthen the lifetime of the restoration, as well as maintaining its remineralizing effect on the remaining structure. ${ }^{5,6}$

Objective: Compare the alteration of the microhardness and surface roughness in the composite resin after the application of three different fluoride varnishes after four weeks.

\section{Material and methods}

The sample consisted of 144 specimens of composite resin. For the preparation of the blocks, cylindrical plastic molds of $5 \mathrm{~mm}$ diameter by $2 \mathrm{~mm}$ in height (3M-ESPE, USA) were used, a celluloid strip and a glass slide were used in the base, then the FILTEK Z350 XT color A2 (3M-ESPE, USA) composite resin was filled with a single increase handled according to the manufacturer's instructions. For the polymerization, the Elipar DeepCure-L was used for 20 seconds according to the manufacturer. The resin specimens were stored in distilled water and placed in an incubator at $37+/-1^{\circ} \mathrm{C}$ for 24 hours. The dry polishing process was done 48 hours later, on both sides for two minutes with the $3 \mathrm{M}$ Shofu system. Later, the blocks were stored in artificial saliva after polishing for 24 hours prior to the application of fluoride varnishes.

\section{Fluoride varnishes application}

It was applied with a microbrush for 1 minute per specimen. Group 1: Duraphat (Colgate-Palmolive), group 2: Clinpro white varnish and group (3M-ESPE, USA) 3: MI varnish (GC), the indications for use according to manufacturer was followed. After the application, the blocks were kept in a glass container with artificial saliva for 7 days, for four consecutive weeks, which was changed daily in order to simulate the oral conditions. The measure on the Vickers 
microhardness test was taken 24 hours after each application. The diagonal measurements of the identations were under a load of $100 \mathrm{~g}$ for 15 seconds. The surface roughness test was the same way as the microhardness; in this case a Mitutoyo rugosimeter was used, and in each reading the instrument traveled a distance of $2.0 \mathrm{~mm}$ (put cut-off) with a speed of $0.5 \mathrm{~mm} / \mathrm{s}$ in one direction, detecting irregularities of the measured surface.

\section{Statistical analysis}

The database was introduced to the statistical program (SPSS version 24). From the data collected, the tables were prepared to evaluate the association between the variables: surface microhardness with fluoride varnish and surface roughness with fluoride varnish. The ANOVA statistical test for repeated samples and Bonferroni was used according to the results of the Kolmogrov-Smirnov normality test.

\section{Results}

The results for the surface microhardness test in the control group were an average of $82.68 \mathrm{~kg} / \mathrm{mm}^{2}$. Compared to the varnishes studied, the sodium fluoride group with CPP-ACP showed lower microhardness in the four weeks application, however the lowest value obtained was in the fourth week with $63.25 \mathrm{~kg} / \mathrm{mm}^{2}$. The fluoride varnish with tricalcium phosphate achieved greater microhardness compared to the two varnishes used. There was no statistically significant difference $(\mathrm{p}=1,000)($ Table 1$)$.

The surface roughness test results for the control group was $0.32 \mathrm{um}$, although the sodium fluoride group show a roughness of a 0.30 um indicating a decrease in the third week, it can be observed in the other results that after each application the values increased in the three different compositions, being the group of sodium fluoride with tri-calcium phosphate the varnish generates greater roughness in the restorative material, showing statistically significant difference in week 1, 2, 3 (Table 2).

Table I Comparison of Surface micro hardness of composite resin after the application of three different fluoride varnishes from week I to 4

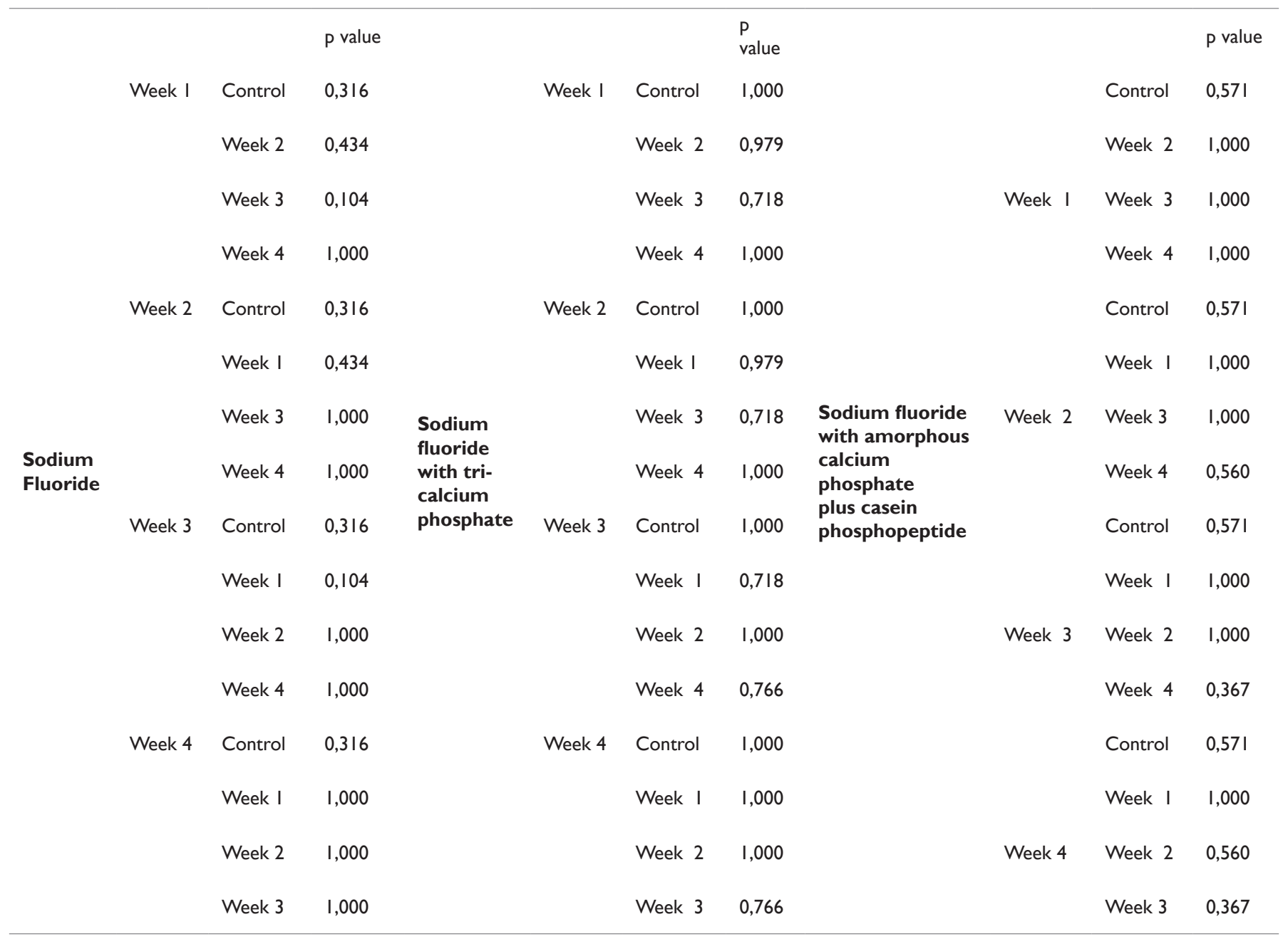

Anova multiple comparisons

Bonferroni

$*_{p}<0.05$ 
Table 2 Comparison of Surface roughness of composite resin after the application of three different fluoride varnish from week I to 4

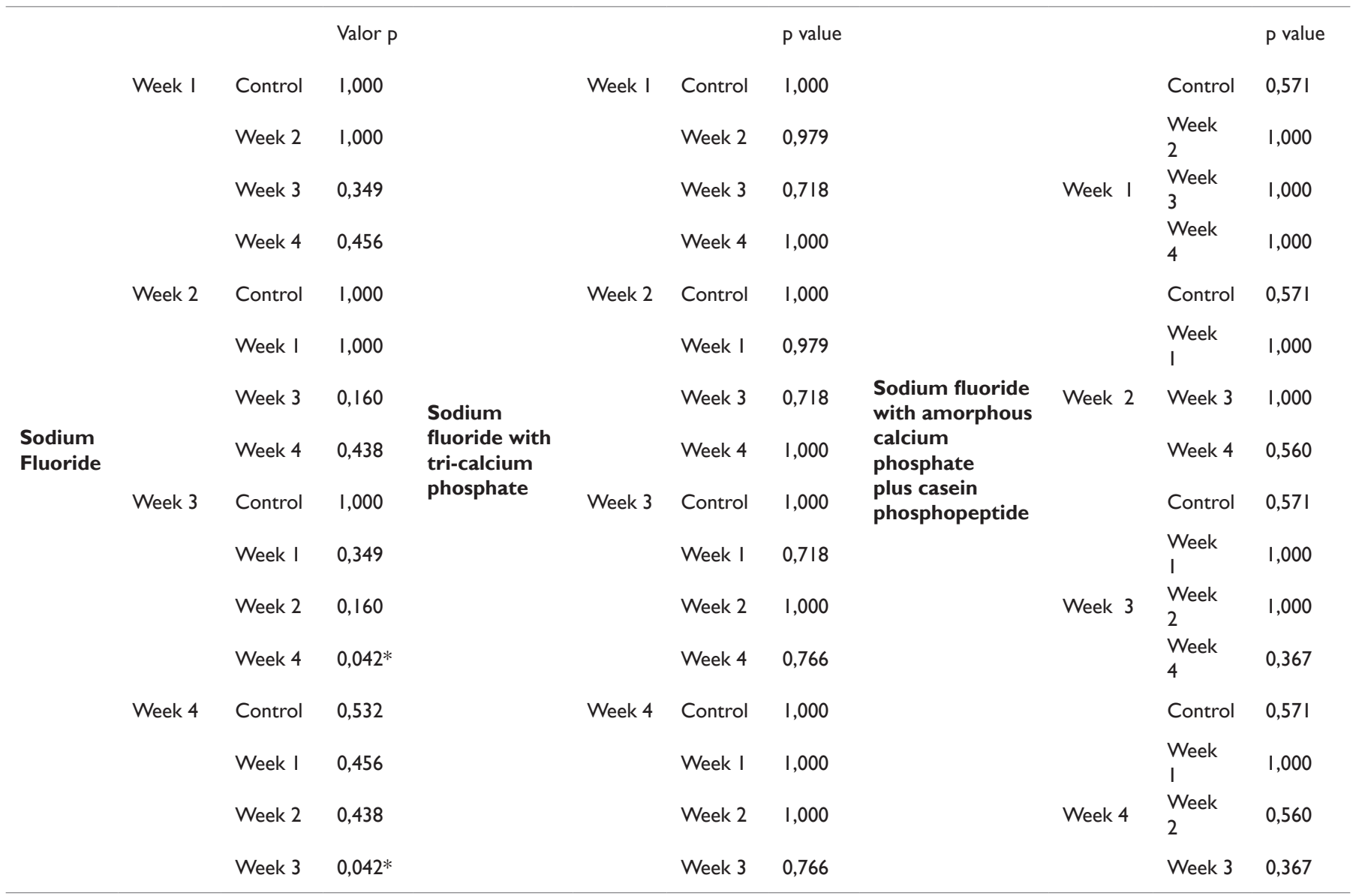

Anova multiple comparisons

Bonferroni

$* \mathrm{p}<0.05$

\section{Discussion}

The values obtained in the present study concluded that microhardness was not statistically different between the three varnishes used, even though that the results showed increased values in week 2 for the fluoride varnish with tri-calcium phosphate. In the study carried out by Doddamani \& Babu, ${ }^{7}$ they found that hardness increases generating greater resistance to demineralization level that can be submitted in the oral cavity. Molaasadolah et al. ${ }^{8}$ corroborates the results obtained by Doddamani, ${ }^{7}$ showing an increase in microhardness after the application of fluorinated varnish. Although the fluoride varnish brands where different in each research.

In the surface roughness studies, statistically significant differences were found with the use of fluoride varnish generating an increase of the surface of the composite, which is consistent with the research carried out by Avsar \& Tuloglu, ${ }^{9}$ they evaluated the effect of acidic and neutral topical fluoride obtaining as a result that both fluorides increase the roughness of the surface, showing higher results for the acid one.

A previous study reported that microhardness decreases after application of a fluoride but increases the surface roughness, which is consistent with the results obtained, because the $\mathrm{pH}$ of the varnish

and gel influences the degradation of the material, which affects the mechanical properties of the surface, in addition it generates more accumulation of plaque causing a decrease in the longevity of the restoration. ${ }^{10}$

\section{Conclusion}

1. There is a variation in the microhardness and roughness on the composite resin after the application of three different fluoride varnishes compositions.

2. The varnishes with sodium fluoride, sodium fluoride and tricalcium phosphate and sodium fluoride with amorphous calcium phosphate plus casein phosphopeptide decrease the surface microhardness of the composite resin.

3. The varnish with sodium fluoride and tri-calcium phosphate generates greater roughness than sodium fluoride and sodium fluoride with amorphous calcium phosphate plus casein phosphopeptide.

\section{Funding details}

Coordination for the Improvement of Higher Education Personnel - Capes. 


\section{Acknowledgements}

None.

\section{Conflicts of interest}

The authors declare that there is no conflict of interest.

\section{References}

1. Dos Santos P, Garcia P, De Oliveira A, et al. Chemical and morphological features of dental composite resin: Influence of light curing units and immersion media. Microsc Res Tech. 2010;73(3):176-781.

2. Voltarelli F, Santos-Daroz C, Alves M, et al. Effect of chemical degradation followed by toothbrushing on the surface roughness of restorative composites. J Appl Oral Sci. 2010;18(6):585-590.

3. Ccahuana V, Ozcan M, Mesquita A, et al. Surface degradation of glass ceramics after exposure to acidulated phosphate uoride. J Appl Oral Sci. 2010;18(2):155-165.

4. Tabatabaei S, SabaghiA. The effect of three mouthwashes on microleakage of a composite resin-an in vitro study. J Am Sci. 2013;9:13-19.
5. Yengopal V, Harneker S, Patel N, et al. Dental fillings for the treatment of caries in the primary dentition. Cochrane Database Systematic Review. 2009;(2):CD004483.

6. Manhart J, Chen H, Hamm G, et al. Review of the clinical survival of direct and indirect restorations in posterior teeth of the permanent dentition. Oper Dent. 2004;29:481-508.

7. Doddamani G, Babu G. Effected of fluoridated varnishes on Surface micro-hardness of enamel. International Journal of Oral Health and Medical Research. 2017;4(2):1-3.

8. Molaasadolah F, Eskandarion S, Ehsani A, et al. In vitro evaluation of enamel microhardness after application of two types of fluoride varnish. $J$ Clin Diagn Res. 2017;11(8):64-66.

9. Avsar A, Tuloglu N. Effect of different topical fluoride applications on the surface roughness of a colored compomer. J Appl Oral Sci. 2010;18(2):171-177.

10. Yeh $\mathrm{S}$, Wang $\mathrm{H}$, Liao $\mathrm{H}$, et al. The roughness, microhardness, and surface analysis of nanocomposites after application of topical fluoride gels. Dental Materials. 2011;27(2):187-196. 